\title{
AMENDMENTS
}

\section{Author Correction: Efficient proximity labeling in living cells and organisms with TurbolD}

Tess C. Branon, Justin A. Bosch, Ariana D. Sanchez, Namrata D. Udeshi, Tanya Svinkina, Steven A. Carr, Jessica L. Feldman, Norbert Perrimon and Alice Y. Ting

Correction to: Nature Biotechnology https://doi.org/10.1038/nbt.4201, published online 20 August 2018.

In the supplementary information for this article originally posted, there were copy-and-paste errors in Supplementary Tables 2-7. In Supplementary Table 2, binary values in tab 1 indicating the presence of a protein in each proteome were incorrect. In Supplementary Table 3, Uniprot accession IDs in tab 1 and binary values in tab 1 indicating the presence of a protein in each proteome were incorrect. In Supplementary Table 4, Uniprot accession IDs and gene names in tabs 1 and 2 were incorrect. In Supplementary Table 5, binary values in tab 4 indicating the presence of a protein in the miniTurbo proteome were incorrect; some gene names were missing in tab 6; and the following columns were incorrect in tab 6: $\log 2(127 \mathrm{~N} / 127 \mathrm{C}), \log 2(128 \mathrm{~N} / 127 \mathrm{C}), \log 2(129 \mathrm{~N} / 129 \mathrm{C}), \log 2(128 \mathrm{C} / 129 \mathrm{C})$, $\log 2(130 \mathrm{~N} / 130 \mathrm{C}), \log 2(131 \mathrm{~N} / 130 \mathrm{C}), \log 2(131 \mathrm{C} / 129 \mathrm{C})$. In Supplementary Table 6, protein names in tabs 1-3 and binary values indicating the presence of a protein in the miniTurbo proteome in tab 4 were incorrect. In Supplementary Table 7, gene names in tab 3 were missing. The errors have been corrected online.

Published online: 20 November 2019

https://doi.org/10.1038/s41587-019-0355-0

๑) The Author(s), under exclusive licence to Springer Nature America, Inc. 2019

\section{Author Correction: Visualizing structure and transitions in high-dimensional biological data}

Kevin R. Moon, David van Dijk (D), Zheng Wang, Scott Gigante (D), Daniel B. Burkhardt (D), William S. Chen, Kristina Yim, Antonia van den Elzen, Matthew J. Hirn (D), Ronald R. Coifman, Natalia B. Ivanova (D), Guy Wolf(D) and Smita Krishnaswamy D

Correction to: Nature Biotechnology https://doi.org/10.1038/s41587-019-0336-3, published online 3 December 2019.

In the version of this article initially published, an affiliation for author Zheng Wang was missing. This affiliation should appear first; it reads "School of Basic Medicine, Qingdao University, Qingdao, China." Also, as a result of a publisher error, the matrix variable $\mathbf{P}$ was replaced by tbfP in equation (4), the matrix variable $\mathbf{Y}$ was formatted italic rather than bold in the first two instances of $\mathbf{Y}_{\text {landmarks }}$, and, in the PDF version, some instances of the variables $\mathbf{P}, \mathbf{x}, \mathbf{y}, \mathbf{p}, \mathbf{B}, \mathbf{J}, \mathbf{Q}, \mathbf{K}, \mathbf{Y}$ and $\mathbf{v}$ were formatted as non-italic plain text instead of non-italic bold. The errors have been corrected in the HTML and PDF versions of the article.

Published online: 2 January 2020

https://doi.org/10.1038/s41587-019-0395-5

() The Author(s), under exclusive licence to Springer Nature America, Inc. 2020 